\title{
Machining of composites by EDM
}

\author{
P. Sreeraj, S. Thirumalai Kumaran, M. Uthayakumar, S. Suresh Kumar
}

\begin{abstract}
Metal matrix composites is a new expansion to cutting-edge materials having astounding properties like a weightless, high explicit quality and great consumption obstruction properties. These types of materials are highly desirable industries. Automobile and aerospace industries require materials that have good strength to weight ratio in order to achieve higher fuel efficiency. It is difficult to machine these types of new generation metal matrix composites with conventional machining techniques. For machining these types of composites now a day's unconventional machining techniques like electrical discharge machining are used. This paper presents review of research work carried out by researchers in EDM on composites. This paper also considers the attribute trends of EDM machining.
\end{abstract}

Keywords-metal matrix, aerospace, specific strength, light weight.

\section{INTRODUCTION}

Electrical discharge machining can be classified into die sinking and wire cut EDM. There are many unconventional machining techniques like ultrasonic machining, water jet machining, abrasive water jet machining is used in industry. But, EDMs are commonly used for machining purpose for creating complex shapes with high accuracy. Experimental set up and specially designed tool for combined EDM and BBM proposed by researchers Yan is shown in Fig 1.

Researches in EDM generally focused on rate of tool wear, material removal rate and surface roughness. Since aluminum metal matrixes are new generation additions which are difficult to machine by conventional methods and for machining this type materials unconventional machining techniques are usually used. Efficiency of EDM machining depends on electrical as well as non-electrical parameters. Nonelectrical parameters include the tool rotation die electric flushing pressure, percentage of addition of reinforcements etc. In order to get good results these parameters must be carefully considered and optimized. Many researchers focused their studies on improvement in MRR, TWR and

Revised Manuscript Received on December 29, 2019.

* Correspondence Author

P. Sreeraj, Department of Automobile Engineering, Kalasalingam Academy of Research and Education, Krishnankoil-626126, India. Email: pathiyasseril@gmail.com

S. Thirumalai Kumaran*, Faculty of Mechanical Engineering, Kalasalingam Academy of Research and Education, Krishnankoil-626126, India. Email: thirumalaikumaran@yahoo.com

M. Uthayakumar, Faculty of Mechanical Engineering, Kalasalingam Academy of Research and Education, Krishnankoil-626126, India. Email: uthaykumar@gmail.com

S. Suresh Kumar, Faculty of Mechanical Engineering, Kalasalingam Academy of Research and Education, Krishnankoil-626126, India. Email: sureshme48@gmail.com surface quality. Actually, EDM machined surface consists of heat affected zone recast or white layer and spattered layer.

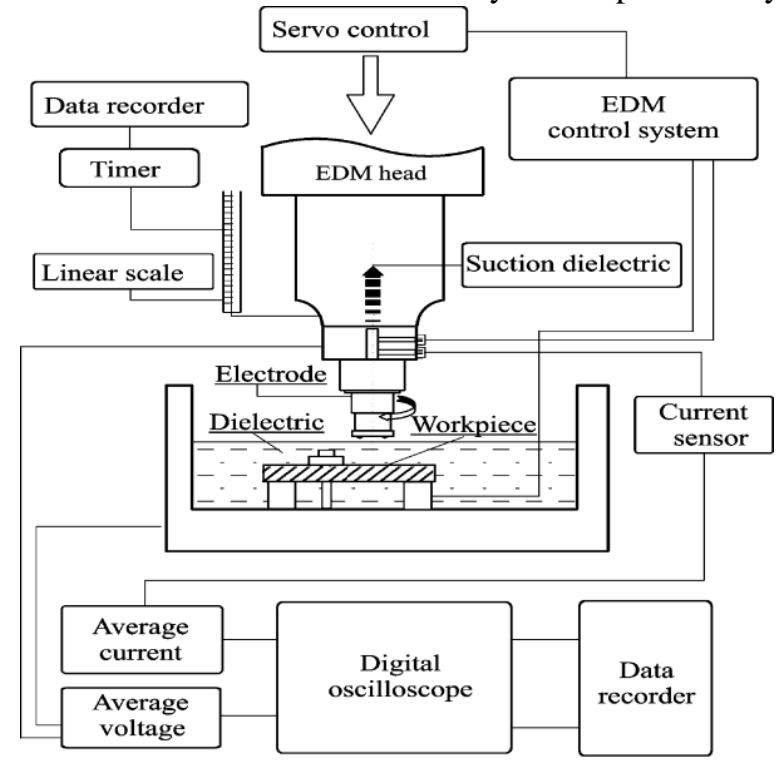

Fig. 1. Experimental set up for combined EDM with BBM

Careful examination of these layers is needed for improvement of machining and different types of layers as shown in Fig 2.

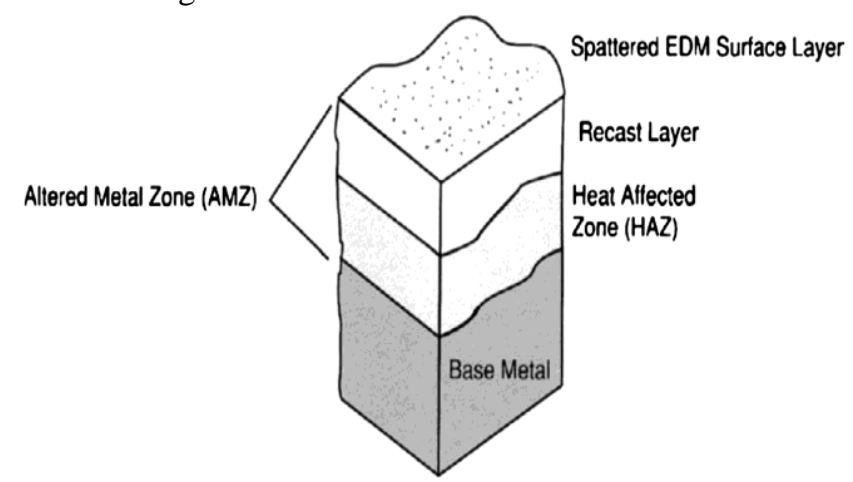

Fig. 2. EDM machined surface

\section{LITERATURE}

Bhaumik et al., [1] EDMed Ti-5Al-2.5Sn compounds with various types of electrodes such as copper brass and zinc. While machining composite with different types of electrodes for analyzing surface roughness $\left(\mathrm{R}_{\mathrm{a}}\right)$, surface fracture density (SFD), radial overcut (RO) and recast layer with different input parameters. It was found that copper electrode was optimal for machining where high accuracy and when good surface finish is required. During EDM process toxic waste and exhaust gases are releases frequently because of high

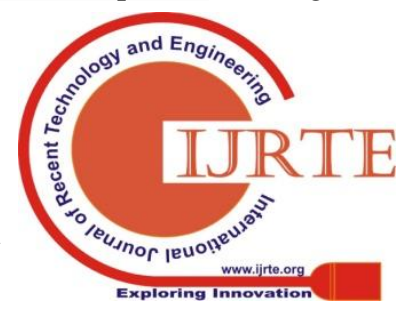




\section{Machining of Composites by Edm}

discharge energies that cause reactions in die electric medium.

Tang et al., [2] In order to overcome this tried tap water as die electric medium while machining $\mathrm{Ti}-6 \mathrm{Al}-4 \mathrm{~V}$ material. Analysis on grey relational is used by efficient of process parameters. It was found that tap water can replace successfully conventional die electric medium.

Agarwal et al., [3] by converting conventional EDM machine to a surface grinding machine for grinding $\mathrm{Al}-10 \mathrm{wt} \% \mathrm{Al}_{2} \mathrm{O}_{3}$ and $\mathrm{Al}-10 \mathrm{wt} \% \mathrm{SiC}$ composite work piece by a diamond grinding wheel. Input parameters like wheel speed, pulse current, depth of cut and work piece speed were analyzed against MRR and surface roughness. experiments proved that there was six times increase in MRR when wheel speed was increased. For preparing a new engineering composite Aluminum 4032 was combined with $\mathrm{Zrb}_{2}$ and Tib2 in various wt. $\%$ at room temperature and the composites was prepared by stir casting method.

Rengasamy et al., [4] EDM machined for optimizing various input parameters against TWR, MRR and surface roughness by Taguchi method. It has proved that by reinforcement mechanical properties increased considerably.

Kumar et al., [5] fabricated aluminum metal matrix composite by sir casting route by reinforcing Aluminum 2618 with AlN (Aluminum Nitride), $\mathrm{ZrB}_{2}$ (Zirconium Boride) and $\mathrm{Si}_{3} \mathrm{~N}_{4}$ (Silicon Nitride) particles. It was found that with increase in reinforcement percentages there is considerable increase in mechanical and other properties. In EDM machining of this prepared composite it was found that MRR and TWR reduced considerably.

Puhan et al., [6] proposed work on power metallurgy process in Aluminum silicon carbide composite and EDM machining of above said composite with commercial grade oil as die electric and copper rod as tool. In this research work in addition to usual input parameters two new parameters such as weight fraction on silicon carbide is compounded and mesh structure was considered against output parameters TWR, MRR, surface roughness and circularity. Process was optimized by a hybrid PCA and fuzzy coupled with Taguchi approach.

Magabe et al., [7] considered the advancement of Ni55.8Ti shape memory alloys while machining with wire-cut EDM. Impacts of procedure parameters, for example, flash hole voltage, beat on-schedule, beat off-time, and wire feed on yield parameters, for example, metal expulsion rate (MRR) and surface unpleasantness. In this procedure Ranking and swarming separation based non-overwhelmed arranging calculation II (NSGA-II) is utilized for procedure improvement. Chakraborty et al., [8] focused on optimizing the process parameters of EDM by Taguchi based utility concept. In this work piece was selected $\mathrm{Al} 6351$ and tool electrode was silicon carbide - copper powder mixed composite tool by powder metallurgy route. Researchers attempted to maximize micro hardness and minimize surface roughness by optimizing the process parameters.

Sidhu et al., [9] prepared SiC/A359 Composite for conducting research on EDM machining. Tool used was electrolytic copper rod of $15 \mathrm{~mm}$ diameter and commercial grade oil was used as die electric in this process. The reactions outputs are all around advanced utilizing attractive quality capacity approach and lexicographic objective programming (LGP) approach.

Singh et al., [10] conducted experiments in EDM machining on composite produced by AA6061reinforced with $20 \%$ alumina $\left(\mathrm{Al}_{2} \mathrm{O}_{3}\right)$ particulates and $1 \% \mathrm{Mg}$ and $2 \%$ $\mathrm{Cu}$ produced by stir casting. Electrolytic copper tool and abrasive powder mixed die electric was used for machining. They optimized the process parameters by Grey relational analysis.

Yan et al., [11] conducted a new method of EDM machining for getting good surface finish by incorporating a rotating copper ring tool embedded with $\mathrm{ZrO}_{2}$ balls. Actually, ring electrode produces electrical discharges for machining and balls which are in contact with the work piece produces a plastic deformation and produce a good surface finish. Central hole in tool sucks the die electric and removes debris from machined area.

Kumar et al., [12] proposed PMEDM method for machining Al-10\%Sicp metal matrix composite prepared through stir casting with copper tool and silicon suspended kerosene as die electric. Conventional Taguchi method was used by researchers for optimizing process parameter for getting high MRR.

Gohil et al., [13] suggested conventional Taguchi and regression analysis for optimization and prediction of process parameters for getting better MRR and better surface finish. Researchers used specially designed rotary tool and titanium alloy (Ti-6Al-4V) as work piece for conducting experiments.

Shihab., [14] used friction-stir-welded 5754 aluminum alloy for cutted with wire EDM for optimizing the process parameters with Box-Behnken design (BBD) of reply surface methods. Analysis of variance was used to find the significant parameters.

Sadagopan et al., [15] conducted experiments with different types of die electrics such as kerosene, biodiesel and transformer oil for investigating the influence of dielectrics in EWR, MRR and surface finish while Electro discharge machining Aluminum Alloy 6063 with copper electrode. It was seen from results that biodiesel as a dielectric has better execution in MRR, EWR and surface completion contrasted and lamp fuel and transformer oil.

Sivaraj et al., [16] This research Proposed on Aluminum and Titanium Carbide powders with different particle sizes mixed and cylindrical composites by power metallurgy method and studies are carried out machining of EDM process. Using a copper tool, the composite was drilled by providing the tool negative polarization. They accomplish investigations to effective parameters such as pulse-on time, current and gap voltage on MRR and TWR for EDM machining. Research proved that material removal rate decreased considerably because of addition on TiC particles and electrode wear increased considerably by current.

Daneshmand et al., [17] used $\mathrm{Al} / 7.5 \% \quad \mathrm{Al}_{2} \mathrm{O}_{3} \quad \mathrm{MMC}$ for EDM machining with 
$\mathrm{Al}_{2} \mathrm{O}_{3}$ mixed kerosene as dielectric. Conventional copper tool was used as a tool in this process. Researchers examined two cases, one with rotary tool and powder mixed die electric and other with stationary tool with powder mixed die electric for marching the composites.

While examining the results it was revealed that rotary tool with powder mixed die electric improved MRR and TWR in a significant manner than other process.

Wang et al., [18] conducted blind-hole drilling of 6061 Al $/ \mathrm{Al}_{2} \mathrm{O}_{3}$ mixed through EDM machining employing non electrical and electrical process parameters. Normal output responses were MRR, TWR and surface roughness and non-electrical parameters includes rotation of tool, injection of flushing pressure of die electrical fluid etc. It was revealed by researchers to electrical parameters has huge consequence on EDM machining.

Mohal et al., [19] produced aluminum metal matrix composites by reinforcing Aluminum alloy 6061 on $10 \%$ silicon carbide particulate (325 mesh size) by stir casting route. Die electric mixed with carbon nano particles used as die electric and copper rod with negative polarity was used as tool electrode. Response surface methodology was used for fabricating regression equation for correlating process parameter with TWR, MRR and surface roughness. It has observed by MRR and TWR improved by using carbon nano particle in mixed die.

Vishwakarma et al., [20] used finite element modeling for evaluating MRR in a powder mixed electric discharge machining of Al-SiC Metal Matrix Composite. Initially FEM model was created for single spark and it was later extended to multi discharge model by extending number of pulses. Temperature distribution model for $\mathrm{Al}-\mathrm{SiC}$ was first evaluated and this is extended for evaluating MRR. Machining of new generation aluminum metal matrix composites which are now a days commonly used in aerospace and defense industries that are difficult to machine compared to machining of conventional metals. Researcher's successfully explored different ways to machine these types of metals in an efficient manner. EDM machining is a successful solution to this. Above section focuses some attempts made by researchers for machining aluminum metal matrix composites by die sinking electrical discharge machining. Even though there are some limitation $s$ to this method such as slow material removal rater high tool wear formation of recast layer and HAZ they devised PMEDM, EDM with ultrasonic vibration and EDM with gas to overcome this inefficiency.

Ramulu et al., [21] fabricated specimens with $15 \mathrm{Vol} \%$ SiCp/Al356-T6 composites. Concave semi cylindrical copper tool was fabricated for conducting experiments. After EDM machining surface texture and fatigue was analyzed and result showed that fatigue strength decreased after EDM machining. Micro cracks, particulate pull out and slight softening of material below the recast layer affected surface integrity. Prediction and optimization of residual stress in machined components is an emerging area of EDM machining. Even though EDM machining is a non-contact process violent sparks causes severe stresses in the machined

component. Careful attention should be given for relieving these stresses is required for long working life of the component. Above section examines the possibility of relieving these stresses and research carried in these areas in past several years.

Lin et al., [22] introduced a new method for improving surface roughness ratio of $\mathrm{Al}-\mathrm{Zn}-\mathrm{Mg}$ alloy through incorporate machining with EDM and ball burnishing machining. Copper tool with ring shape was used a tool for this machining. $\mathrm{ZrO}_{2}$ balls were joined to the tool electrode in this experiment for providing burnishing. From results researchers came to conclusion that considerable improvement in quality of machining.

Kansal et al., [23] used commercially available kerosene for suspending aluminum powder to make powder suspended dielectric. $\mathrm{Al}-10 \% \mathrm{SiCP}$ has utilized as work piece and copper rod as used as tool. Second order regression equations were made correlate surface roughens and MRR with process parameters. Optimal conditions were found by Taguchi, method and conformity test are conducted to validate the experiment. Solution revealed that powder mixed dielectric could provide better surface integrity.

\section{SCOPE OF WORK}

In this paper brief discussion proposed on the EDM process by using aluminum metal matrix composites (AMMCs). From this discussion, the following steps are

1. From the above analysis it is clear that most of the study in EDM machining of MMCS was confined to machining of aluminum meat matrix composites.

2. EDM is a multi-criteria machining process so optimization and modelling are a complicated process. Forming and creating mathematical models relating to this is a Himalayan task. New generation methods like ANN, GA, SA and fuzzy logic are effectively applied to execute this multi objective optimization issues.

3. To sudden heating and cooling in EDM machining residual stresses are created. This residual stress is harmful to the machined component because early failure of component is expected. Study of this residual stresses is new research area in EDM machining.

4. In most of the EDM process copper, electrolyte copper and graphite tools are used. There is a chance for composite tool.

5. Non electrical parameters are tool rotation vibration on tool and blushing pressure which affects the performance of EDM process.

6. Hybrid machining techniques are another research area to be focused in improving the efficiency of EDM process.

7. Use of water as die electric is a new emerging area where research attention to be focused.

8. There is very little research work conducted in ultra-sonic assisted EDM with gas as die electric. So, there is immense chance for scope for future research.

\section{REFERENCES}

1. Munmun Bhaumik, Kalipada Maity, "Effect of Electrode 
Materials on Different EDM Aspects of Titanium Alloy," Silicon, Feb 2019, pp 1-10.

2. L. Tang and Y. T. Du, "Experimental study on green electrical discharge machining in tap water of $\mathrm{Ti}-6 \mathrm{Al}-4 \mathrm{~V}$ and parameters optimization," International Journal of Advanced Manufacturing Technology, Volume: 70, Jan 2014, pp: 469-475.

3. Shyam Sunder Agrawal a \& Vinod Yadava, "Modeling and Prediction of Material Removal Rate and Surface Roughness in Surface-Electrical Discharge Diamond Grinding Process of Metal Matrix," Composites, Materials and Manufacturing Processes, Volume 28, Issue 4, 2013 pp:381-389.

4. N.V.Rengasamy, M.Rajkumar, S. Senthil Kumaran, "An Analysis of Mechanical Properties and Optimization of EDM Process Parameters of Al 4032 Alloy Reinforced with $\mathrm{Zrb}_{2}$ and $\mathrm{Tib}_{2}$ In-Situ Composites,' Journal of Alloys and Compounds, Volume 662, 2016, pp. 325-338.

5. N.Mathan Kumar ,S. Senthil Kumaran and L.A. Kumaraswamidhas, "An investigation of mechanical properties and material removal rate, tool wear rate in EDM machining process of AL2618 alloy reinforced with $\mathrm{Si}_{3} \mathrm{~N}_{4}$, AlN and $\mathrm{ZrB}_{2}$ composites," Journal of Alloys and Compounds, Volume 650, 2015, pp. 318-327.

6. Debaprasanna Puhan, Siba Sankar Mahapatra, Jambeswar Sahu, Layatitdev Das, "A hybrid approach for multi-response optimization of non-conventional machining on AlSiCp MMC," Measurement, Volume 46, Issue 9, 2013, pp. 3581-3592.

7. Raymond Magabe, Neeraj Sharma, Kapil Gupta and J Paulo Davim, "Modeling and optimization of Wire-EDM parameters for machining of Ni55.8Ti shape memory alloy using hybrid approach of Taguchi and NSGA-II," The International Journal of Advanced Manufacturing Technology, Vol - 102, 2019, pp: 1703-1717.

8. N. Pallavi Senapati, Rawnak Kumar, S. Tripathy and Amruta Rout, "Multi-objective Optimization of EDM Process Parameters Using PCA and TOPSIS Method During the Machining of Al-20\% SiCp Metal Matrix Composite," Innovative Design and Development Practices in Aerospace and Automotive Engineering, 2016, pp 359-367.

9. Sarabjeet Singh Sidhu, Morteza Yazdani, "Comparative Analysis of MCDM Techniques for EDM of SiC/A359 Composite," Arabian Journal for Science and Engineering, Vol : 43, Issue: 3, 2017, pp. 1-10.

10. S. Singh and M.F Yeh, "Optimization of Abrasive Powder Mixed EDM of Aluminum Matrix Composites with Multiple Responses Using Gray Relational Analysis," Journal of Materials Engineering and Performance, Volume 21, Issue 4, , 2012, pp. 481-491.

11. B.H. Yan, Y.C. Lin, F.Y. Huang "Surface modification of Al-Zn-Mg alloy by combined electrical discharge machining with ball burnish machining," International Journal of Machine Tools and Manufacture, Volume 42, Issue 8, 2002, pp. 925-934.

12. J. Harmesh Kumar, Paulo Davim, "Role of Powder in the Machining of Al-10\%Sicp Metal Matrix Composites by Powder Mixed Electric Discharge Machining," Journal of Composite Materials, Volume: 45 issue: 2, 2010, pp. 133-151.

13. Vikas Gohil and Yogesh M Puri, "Statistical analysis of material removal rate and surface roughness in electrical discharge turning of titanium alloy (Ti-6Al-4V)," Proceedings of the Institution of Mechanical Engineers, Part B: Journal of Engineering Manufacture, 2016, pp. 1-12.

14. Suha K. Shihab, Optimization of "WEDM Process Parameters for Machining of Friction-Stir-Welded 5754 Aluminum Alloy Using Box-Behnken Design of RSM," Arabian Journal for Science and Engineering, Vol: 43, Sep-2018, pp:5017-5027.

15. P.Sadagopan and B.Mouliprasanth, "Investigation on the influence of different types of dielectrics in electrical discharge machining," The International Journal of Advanced Manufacturing Technology, Volume 92, Issue 1-4, 2017, pp. 277-291.

16. M. Sivaraj and N. Selvakumar, "Experimental Analysis of Al-TiC Sintered Nano Composite on EDM Process Parameters Using ANOVA," Materials and Manufacturing Processes, Volume 31, Issue 6, 2016, pp:802-812.

17. Saeed Daneshmand, Behnam Masoudi and Vahid Monfared, "Electrical discharge machining of $\mathrm{Al} / 7.5 \%$ al2o3 $\mathrm{MMCs}$ using rotary tool and $\mathrm{Al}_{2} \mathrm{O}_{3}$ powder" Surface review and letters, vol. 24, 2017.

18. Che Chung Wang and Biing HwaYan "Blind-hole drilling of $\mathrm{Al}_{2} \mathrm{O}_{3} / 6061 \mathrm{Al}$ composite using rotary electro-discharge machining," Journal of Materials Processing Technology, Volume 102, Issues 1-3, 2000, pp. 90-102.

19. Sachin Mohal and Harmesh Kumar, "Parametric Optimization of Multiwalled Carbon Nano tube Assisted Electric Discharge Machining of Al-10\%SiCp Metal Matrix Composite by Response Surface Methodology," Materials and Manufacturing Processes, Vol-32, 2017, pp:263-273.

20. U K. Vishwakarma, A. Dvivedi, P. Kumar, "Finite Element Modeling of Material Removal Rate in Powder Mixed Electric Discharge Machining
S. Thirumalai Kumaran completed his Ph.D. degree in Mechanical Engineering from Kalasalingam Academy of Research and Education (KARE), India, in 2015. After completing Ph.D., he worked as an Assistant Professor at School of Mechanical Engineering, Yeungnam University, South Korea for a period of one year. He is currently an Associate Professor at School of Automotive and Mechanical Engineering, KARE.

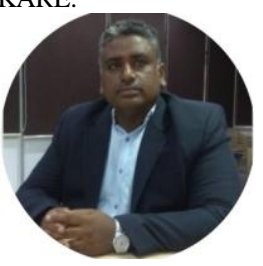

M. Uthayakumar completed his Doctoral work in the area of Precision Machining. He is actively working in the development of high strength light weight composites. His other areas of interest include processing of metal matrix, polymer matrix, ceramic matrix composite and its performance studies.

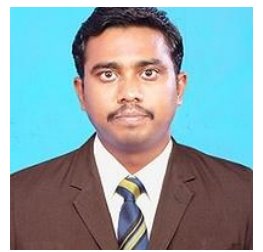

S. Suresh Kumar completed his Ph.D. degree in Mechanical Engineering from Kalasalingam Academy of Research and Education (KARE), India in 2016. He also completed Post-doc from Karadeniz Technical University, Turkey in 2018 Currently, he is doing his research in the area of non-traditional machining on metal matrix composites. 